\title{
Suspecting Airway Foreign Body in Agenesis of the Lung: A Rare Incidence of Misdiagnosis
}

\author{
${ }^{1}$ Santosh Kumar Swain, ${ }^{2}$ Sidarth Mohanty, ${ }^{3}$ Neha Singh, ${ }^{4}$ Jashashree Choudhury, ${ }^{5}$ Mahesh Chandra Sahu
}

\begin{abstract}
Agenesis of the lung is an extremely rare condition. Suspecting foreign body in agenesis of the lung is a diagnostic dilemma. We report the case of an 11 months old boy who presented with cough and cold; examination showed decreased air entry on the right side and investigations reported collapse on chest X-ray and plain computed tomography (CT) scan of thorax. Pediatrician suspected airway foreign body. Rigid bronchoscopy was done to confirm the diagnosis, but there was blind end in the right bronchus with no foreign body seen. Contrast-enhanced CT scan (CECT) of the neck and chest confirmed the aplasia of right lung. Agenesis of the lung should be kept in the mind by the clinician when dealing with foreign body in airway. This can prevent unnecessary intervention like rigid bronchoscopy.
\end{abstract}

Keywords: Agenesis of the lung, Airway foreign body, Bronchoscopy.

How to cite this article: Swain SK, Mohanty S, Singh N, Choudhury J, Sahu MC. Suspecting Airway Foreign Body in Agenesis of the Lung: A Rare Incidence of Misdiagnosis. Int $J$ Phonosurg Laryngol 2015;5(1):32-34.

Source of support: Nil

Conflict of interest: None

\section{INTRODUCTION}

Foreign body (FB) inhalation is a clinical emergency requiring prompt action to ensure speedy recovery and minimizing of complications. Inhalation of FB is the most common cause of accidental death in children under the age of 1 year. ${ }^{1}$ The risk of airway FB remains very high up to age 3 years. Prevention and early diagnosis can be lifesaving. Aspirated foreign bodies in the airway continue

\footnotetext{
${ }^{1,4}$ Associate Professor, ${ }^{2}$ Professor

${ }^{3}$ Assistant Professor, ${ }^{5}$ Research Associate

${ }^{1}$ Department of ENT, Institute of Medical Sciences and Sum Hospital, Bhubaneswar, Odisha, India

${ }^{2,3}$ Department of Anesthesia, Institute of Medical Sciences and Sum Hospital, Bhubaneswar, Odisha, India

${ }^{4}$ Department of Pediatrics, Institute of Medical Sciences and Sum Hospital, Bhubaneswar, Odisha, India

${ }^{5}$ Department of Central Research Laboratory, Institute of Medical Sciences and Sum Hospital, Bhubaneswar, Odisha, India

Corresponding Author: Santosh Kumar Swain, Associate Professor, Department of ENT, Institute of Medical Sciences and Sum Hospital, Bhubaneswar, Odisha, India, Phone: 06742386292 e-mail: santoshvoltaire@yahoo.co.in
}

to provide challenges to the otorhinolaryngologist. The diagnosis can be missed even by an experienced clinician. This case was initially diagnosed as FB by pediatrician due to decreased or absent air entry on one side of the chest during management of his cold and cough. More often, FB in the airway is suspected from chest X-ray after reporting as collapse in same side of decreased air entry. The rigid bronchoscopy and contrast CT scan of the neck and chest confirmed the diagnosis of agenesis of the lung in the same side. Agenesis of the lung is an extremely rare congenital anomaly with a reported prevalence of 34 per 10 lakhs live births. ${ }^{2}$ Autosomal recessive may be the mode of inheritance and consanguinity has been reported. ${ }^{3}$ The first case from India was reported at autopsy in 1923. ${ }^{4}$ Our case was interestingly misdiagnosed as airway FB initially and confirmed as agenesis of the lung in bronchoscopy with computed tomography (CT) scan of the neck and thorax with contrast.

\section{CASE REPORT}

An 11 months old male child came to pediatric OPD with complaints of cough and cold since 5 days. The baby had no breathing difficulty. Except cough, the child had no other clinical manifestations. On auscultation, there was decreased air entry into right lung. X-ray chest showed collapse of the lungs in the same side (Fig. 1). After suspecting FB in airway, pediatrician sent the child to the ENT out-patient department (OPD). There was no respiratory distress or cyanosis. Flexible laryngoscopy was done but there was no FB seen in larynx or upper part of trachea. A plain CT scan of the chest revealed collapse of the right lung with hyperinflation of left lung to right side (Figs 2 and 3).

Laboratory investigations were normal. To rule out FB airway, patient was shifted to ENT operation theater for rigid bronchoscopy under general anesthesia. The rigid ventilating type of bronchoscope with venturi connection was used. Then, with the help of rigid bronchoscope ( $3 \mathrm{~mm}$ size), FB was searched for in trachea and lower airway. However, no FB was seen. To our surprise, we did not enter into the right side bronchus and bronchioles and nothing was visible on the right side. Bronchoscopy revealed obliteration of the right bronchus just after carina. With doubt in mind, we planned for contrast 


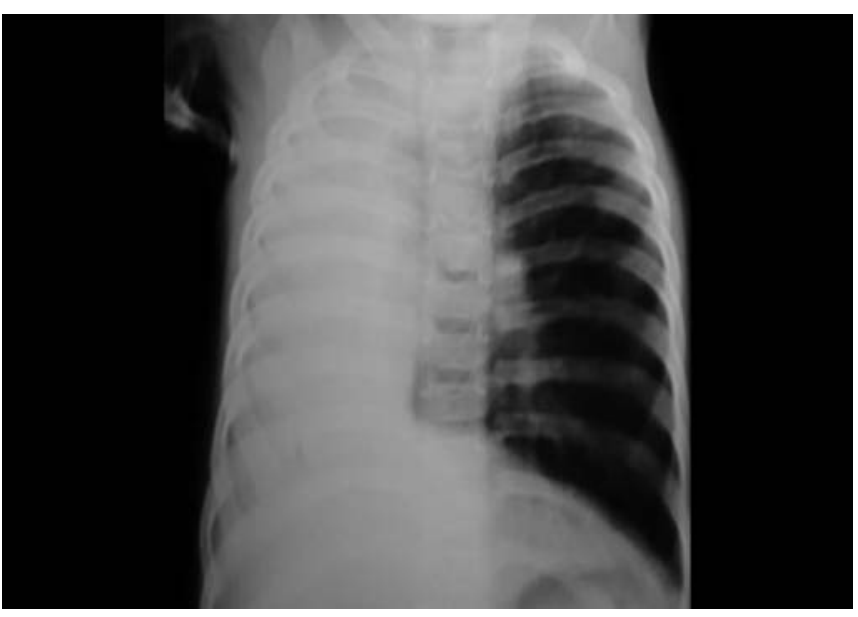

Fig. 1: Plain chest X-ray showing collapse of the right lung

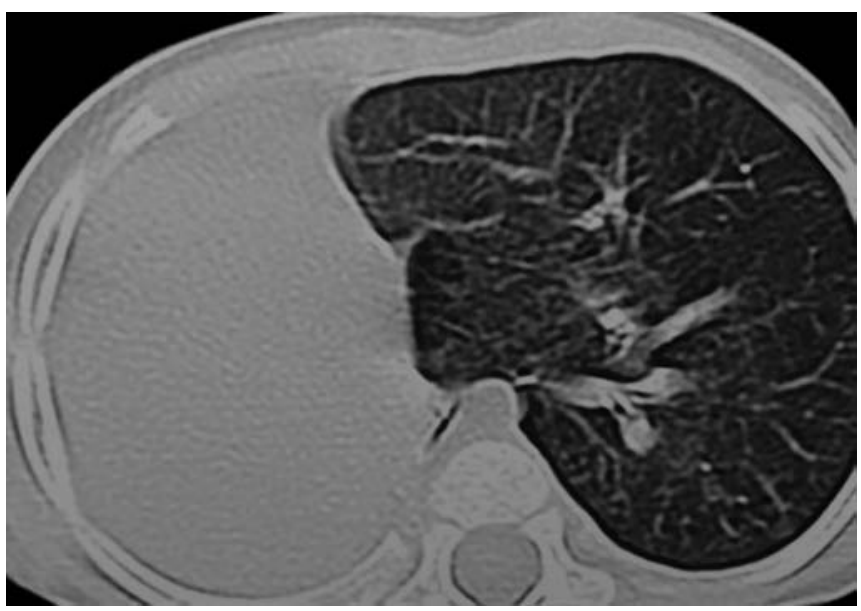

Fig. 3: Plain CT scan of thorax (Axial view) showing absence of the right lung

CT scan of chest after recovery of the child. This was reported as congenital agenesis of the right lung and hyper inflation of left lung to the right side (Figs 4 and 5). Electrocardiogram and ultrasonography of the abdomen were normal.

\section{DISCUSSION}

Inhalation of FB in a child can be serious and potentially, fatal. Early intervention is vital for to save the life.

Sometimes, aspiration of FB creates a diagnostic dilemma as in our case of agenesis of lung. In the diagnosis of FB aspiration in the airway, the history has a significant role. In our case, there was no history of FB aspiration except the sudden onset of cold and cough with abnormal chest findings. Symptoms of sudden onset of cough, dyspnea and respiratory distress, especially in children, should alert the physician for the suspicion of FB aspiration. ${ }^{5}$ The indications for bronchoscopy in inhaled FB are not well defined. Usually, a clear history of sudden suffocation with abnormal chest examination, or radiologic findings are accepted as reliable indication for

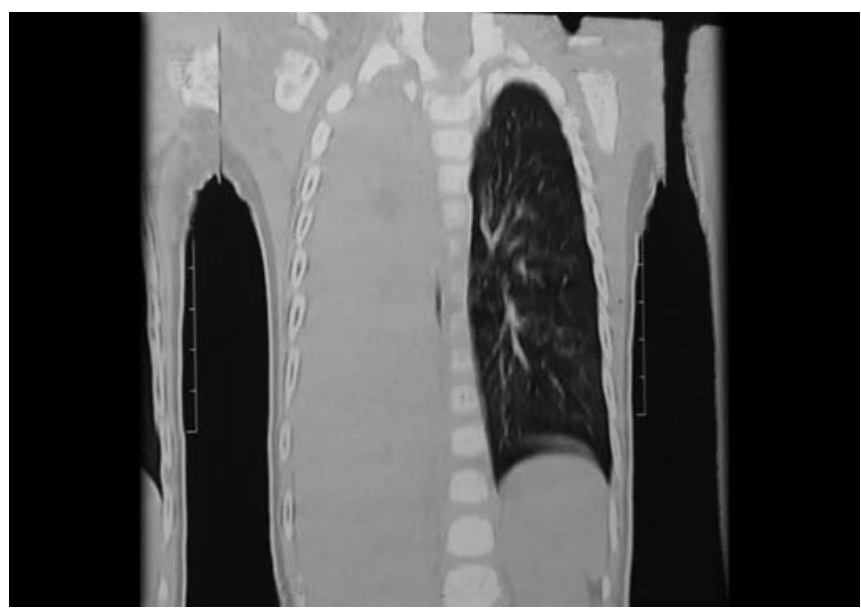

Fig. 2: Plain CT scan of thorax (coronal view) showing absence of the right lung with hyperinflation of left lung to right side

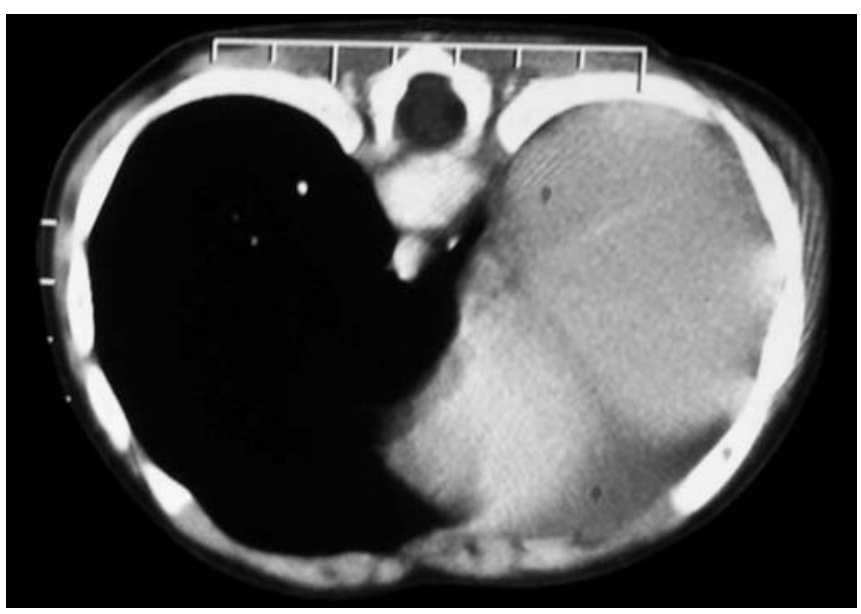

Fig. 4: Contrast CT scan of thorax (Axial view) showing absence of the right lung with mediastinal shift to the left

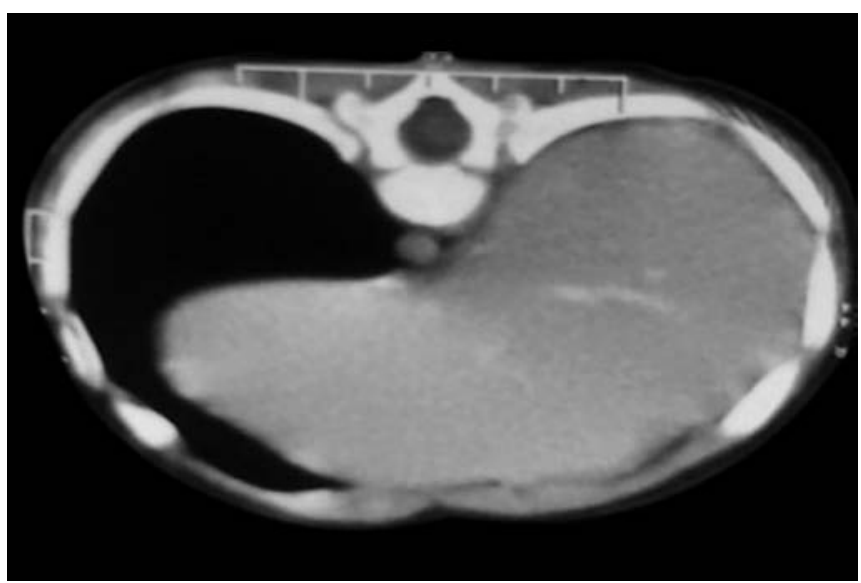

Fig. 5: Contrast CT scan of thorax (Axial view) showing absence of the right lung with hyperinflation of left lung to the right side

performing bronchoscopy. ${ }^{6}$ In our case, the X-ray findings of collapse of one lung with decreased air entry on the same side were warranted for bronchoscopy to rule out FB. This showed no FB; instead, there was blind end in the right bronchus. The findings were in favor of agenesis of the lung, which was confirmed by CECT. There was congenital absence of the lung on the right side. 
Agenesis of lung is due to failure of development of the primitive lung bud. This condition was first discovered accidentally at the autopsy of an adult female in 1673 by De Pozze. ${ }^{7}$ Patients with one lung have been reported to survive well into adulthood without much complaint. ${ }^{8}$ The oldest patient cited by Oyamada et al was 72 years old. ${ }^{9}$ No significant etiological factors have been found in patients, and thus genetic, teratogenic and mechanical factors have been responsible. ${ }^{10}$ Chronic respiratory failure and eventually respiratory infections can develop in later part of life. ${ }^{11}$ In unilateral lung agenesis, the trachea continues directly into the main bronchus of the normally developed lung and respiratory distress commonly occur due to retention of bronchial secretions and inflammation. ${ }^{12}$ Many times, the patient may be asymptomatic and the diagnosis is suspected from radiological tests, like chest $\mathrm{X}$-ray or detected during autopsy. Chest skiagram, bronchoscopy, bronchography and angiography are needed for diagnosis of pulmonary agenesis. With the onset of CT scan, these invasive techniques which entail significant risk have become unnecessary. ${ }^{13}$ Computed tomography of the chest reveals opaque hemithorax with mediastinal shift toward the left side and bony cage symmetry. Computed tomography scan also delineates lung parenchyma with pulmonary and bronchial tree. This case was initially suspected with FB in the airway. The diagnosis was done by a local pediatrician on seeing the decreased air entry in right side of the lung and collapse of lung in X-ray. Inhalation of FB can result in sudden suffocation, choking, paroxysms of coughing followed by tachypnea and dyspnea. Symptoms produced by airway $\mathrm{FB}$ vary with their size, composition, location and duration. In our case, the child had minimal signs and symptoms, i.e. cold and cough. In view of our findings, we recommend that rigid bronchoscopy can be performed as the first procedure in children with one of the following findings; decreased lung sounds, unilateral atelectasis or hyperinflation or mediastinal shift or a radio-opaque foreign body. Another indication for immediate rigid bronchoscopy is to treat an asphyxiating child with a history of suspected FB inhalation. The main goal in treating FB inhalation in children is prevention. Since aspiration of foreign bodies can be a serious and sometimes fatal problem, early intervention and proper management is vital. A careful history and clinical examination are strong indicators of the diagnosis and raise the index of suspicion of an aspirated FB. Even though agenesis of the lung is extremely rare, it should be in the mind when dealing with FB airway.

\section{CONCLUSION}

Inhalation of FB is a preventable mishap and sometimes misdiagnosed in certain situation which may need urgent bronchoscopy. In pediatric patients, history and clinical examination are equally, if not more, important than investigations. A dilemma arises when a child presents with decreased air entry in one side of the lung with no history of FB entry. Agenesis of the lung should be in the mind of the clinician when dealing with foreign body in airway which can prevent intervention, like rigid bronchoscopy.

\section{ACKNOWLEDGMENT}

Authors would like to thank Prof RN Samal, Head, Department of Otorhinolaryngology and Prof RN Padhy, Head, Central Research Laboratory, IMS and Sum Hospital, SOA University, Bhubaneswar, Odisha, India for drafting the article.

\section{REFERENCES}

1. Mofenson HC, Greensher J. Management of the choking child. Pediatr Clin North Am 1985;32(1):183-192.

2. Borja AR, Ransdell HTJ, Villa S. Congenital development arrest of the lung. Am Thorac Surg 1970;10(4):317-326.

3. Hastings R, Harding D, Donaldson A, Leibling R, Hayes A, Kraus A, Joss S, Narayanaswamy S, Turnpenny P, Smithson S. Mardini-Nyahan association (lung agenesis, congenital heart, and thumb anomaly): three new cases and possible recurrence in a sib - is there a distinct recessive syndrome. Am J Med Genet A 2009;149A(12):2838-2842.

4. Muhamed KSN. Absence of left lung. Indian Med Gaz 1923; 58(1):262-264.

5. Aydogan LB, Ulku T, Levent S, Mete K, Can O. Rigid bronchoscopy for the suspicion of foreign body in the airway. Int J Pediatric Otorhinolaryngology 2006;70(5):823-828.

6. Shlomo C, Avraham A, Simon G, Menachem G, Eitan K, Chaim S. Suspected foreign body inhalation in children: What are the indications for Bronchoscopy? The J Pediatric 2009;155(2):276-280.

7. De Pozze, Brescia MA, Amermon EE, Sharma KK. Agenesis of the Left Lung. Arch Pediatric 1960;77:485-490.

8. Kisku KH, Panigrahi MK, Sudhakar R, Nagarajan A, Ravikumar R, Daniel JR. Agenesis of lung-a reported of two cases. Lung India 2008;25(1):28-30.

9. Oymada A, Gasul BM, Holinger PM. Agenesis of the lung. Am J Dis Child 1953;85(2):182-187.

10. Yetim TD, Bayarogullari H, Yalcin HP, Arica V, Arica SG. Congenital agenesis of the left lung: A rare case. J Clin Imaging Sci 2011;1:47.

11. Viora E, Sciarrone A, Bastonero S, Errante G, Campogrande M. Parental diagnosis of isolated unilateral pulmonary agenesis in the second trimester. Ultrasound Obstet Gynecol 2002;19(2):206-207.

12. Krivchenya TD, Khursin VN, Lysak CV. Aplasia of the right lung in a 4-year old child: Surgical stabilization of the mediastinum by diaphragm translocation leading to complete recovery from respiratory distress syndrome. J Pediatr Surg 2000;35(10):1459-1502.

13. Bhagat R, Panchal N, Shah A. Pulmonary Aplasia: A CT appearance. Ind Pediatr 1992;29:1410-1412. 